\title{
THE MARTIAN SATELLITES
}

\author{
S. FRED SINGER \\ Department of the Interior
}

The Martian satellites are some of the most interesting and accessible objects in the solar system, and therefore worth studying or even visiting. As small bodies, on the order of kilometers in diameter (see table I), they may have experienced no internal heating or volcanism, and could therefore be original condensations in the solar system. They may thus be the only remnants of the original planetesimals.

An additional point of interest is that they are the only satellites in the inner part of the solar system-Earth's Moon is generally assumed to be a sister planet. As such, their study could illuminate one of the important differences between the inner and outer parts of the solar system.

Let us assume first that Phobos and Deimos were formed when Mars was formed, that they constitute original planetesimal material, that their orbits have not changed. The orbits are nearly circular and equatorial, like those of many satellites of the outer planets. Kuiper (1956) has also pointed to certain regular relations between satellite masses and distances.

However, we note some peculiarities. First, that Deimos is located at 6.9 Martian radii, which is just beyond the synchronous orbit limit of Mars. We also note that Phobos is located at 2.8 Martian radii, which is just near the Roche limit.

We may therefore consider the alternative possibility that Phobos and Deimos are captured planetesimals, asteroids (i.e., fragments of larger bodies), or captured cometary nuclei. Any of these three possibilities would give us a valid reason for direct examination of the Martian satellites. But how were they captured? Clearly not in an initially dense atmosphere that then conveniently disappeared. This would not explain why the inclinations are so close to zero. This leaves tidal capture followed by tidal evolution as a distinct possibility.

If we start with the present orbits and calculate the orbits backward in time using a tidal perturbation in which the bulge position depends in magnitude and sign on the relative angular velocity of the satellite and of Mars, we obtain results that suggest that both Phobos and Deimos started with highly eccentric orbits and might have been captured (Singer, 1968). It is interesting to note in figure 1 that the orbital evolution of an original parabolic orbit can proceed 


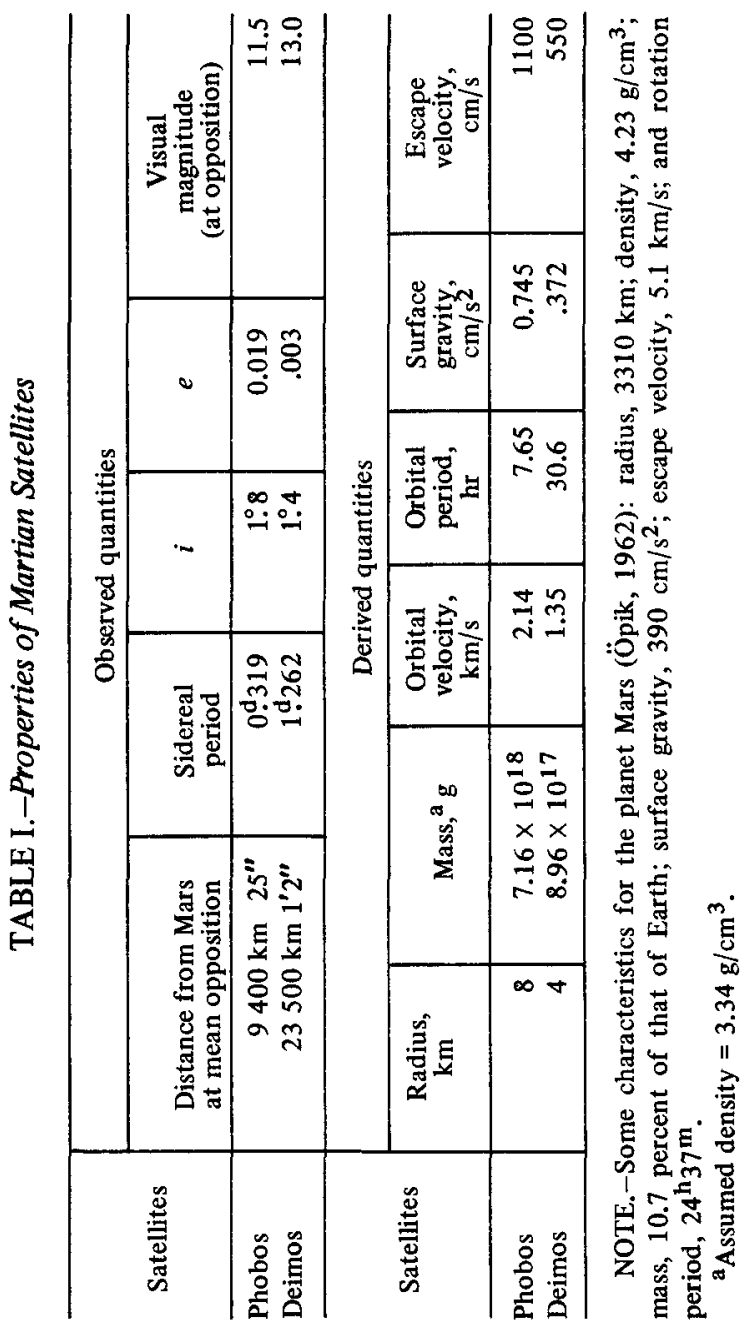




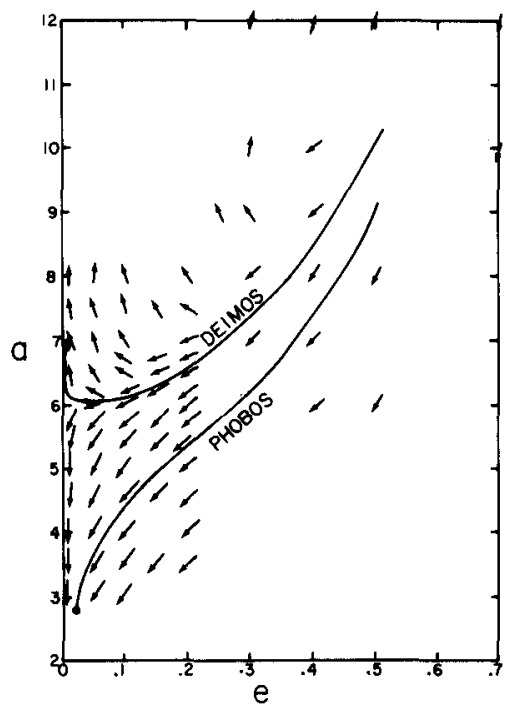

Figure 1.-Orbit evolution, due to tidal forces, of the Martian satellites Phobos and Deimos plotted in $a, e$ space. At present, Phobos has a semimajor axis of 2.8 Mars radii and an eccentricity of 0.019 and Deimos has a semimajor axis of $6.9 \mathrm{Mars}$ radii and an eccentricity of 0.003 . The arrows are plotted so that $\tan \theta=(d a / d t) /(d e / d t)$. Starting with orbits of large $a$ and $e$, evolution proceeds toward smaller values. An interesting bifurcation phenomenon develops leading to satellites of the Deimos class spiraling outward and of the Phobos class spiraling inward. The calculations were performed to illustrate an extension of classical tidal theory in which dissipation is taken to be frequency dependent.

either toward a Phobos-type orbit, which must then shrink into the planet, or toward a Deimos-type orbit, which will be permanently captured.

Unfortunately, however, tidal capture is quite improbable for objects of the small mass of Deimos and Phobos, and, further, the time scale of evolution is of the order of 30 billion $\mathrm{yr}$ and therefore much too long. One way of overcoming these objections would be to assume extremely large internal dissipation for Mars, but that does not seem realistic. Or we could assume that Phobos and Deimos were initially much more massive objects and therefore more easily captured, and that they lost their mass gradually or more recently. Perhaps the most likely explanation is that Mars captured into a prograde orbit a large object, either a comet or a solid object, that through tidal evolution soon crashed into the surface of Mars. But before the crash, Phobos and Deimos were split off and they still survive. Phobos, however, may not survive for much more than 10 million yr.

This suggestion would solve the capture problem and time-scale problem as well. More calculations are needed and will be carried out in the near future. A great advantage of this explanation is that it is consistent with an origin of 
Earth's Moon by capture (Singer, 1968), and also with the nearly zero angular momentum of Venus explained by capture of a moon into a retrograde orbit (Singer, 1970). In general, these suggestions fit in well with Urey's picture of the existence of many moon-sized bodies in the inner solar system (Urey, 1952).

One might like to verify the suggestion advanced here in three different ways: (1) by direct examination of the satellites, (2) by looking for subvisual satellites, and (3) by examining the Martian surface for unusual craters in the equatorial plane that are remnants of the original big object that crashed.

\section{REFERENCES}

Kuiper, G. P. 1956, On the Origin of the Satellites and the Trojans. Vistas in Astronomy (ed., Arthur Beer), vol. 2, pp. 1631-1666. Pergamon Press. New York.

Öpik, E. J. 1962, Atmosphere and Surface Properties of Mars and Venus. Progress in Astronautical Sciences (ed., S. F. Singer), vol. 1, pp. 261-342. North-Holland Pub. Co. Amsterdam.

Singer, S. F. 1968, The Origin of the Moon and Geophysical Consequences. Geophys. J. Roy. Astron. Soc. 15, 205-226.

Singer, S. F. 1970, How Did Venus Lose Its Angular Momentum? Science 170, 1196-1198.

Urey, H. C. 1952, The Planets: Their Origin and Development. Yale Univ. Press. New Haven, Conn.

\section{DISCUSSION}

KUIPER: There was a meeting in 1953 where I gave a paper (Kuiper, 1956) on the universal relation of satellite masses in terms of the primary. This relation is of such a nature that the Mars satellites have exactly the right masses for their distance. Therefore, it was my conclusion that the close satellites of Mars, Jupiter, and Saturn were formed essentially where they are now. These systems have not been appreciably disturbed. I think it is out of the question that these are captured asteroids; there is no relation between the two.

BANDERMANN: How do you explain the very short lifetime of Phobos?

KUIPER: The notion that Phobos has a short lifetime was based on erroneous observations at the Naval Observatory some 20 yr ago.

KAULA: Pertinent to the lifetime problem of Phobos, it seems extremely probable that the tidal evolution has been hung up at various stages at commensurabilities with longitudinal variations in the Martian gravitational field. The torque exerted by the tidal bulge of such a tiny satellite is plausibly much less than the torque by the fixed irregularities in the Martian mass distribution:

$$
\frac{m}{r} \frac{\partial U_{T}}{\partial \lambda}<\frac{m}{r} \frac{\partial U_{l m p q}}{\partial \lambda} \quad \text { or } \quad U_{T}<U_{l m p q}
$$

where $m, r$, and $\lambda$ are Phobos' mass, radial coordinate, and longitude, and the potential functions are

$$
U_{T}=k G \frac{m R^{5}}{r^{6}} \times \frac{3}{4} \sin 2 \delta T
$$

$U_{l m p q}=\frac{G M^{*}}{a}\left(\frac{R}{a}\right)^{l} F_{l m p}(i) G_{l p q}(e) J_{l m} \cos \left[(l-2 p) \omega+(l-2 p+q) M+m\left(\Omega-\theta-\lambda_{l m}\right)\right]$ 
where $a, e, i, \omega, \Omega$, and $M$ are Kepler elements; $M^{*}, R$, and $\theta$ are mass, radius, and the reference meridian sidereal time of Mars; $k$ is the tidal Love number of Mars; $G$ is the gravitational constant; $J_{l m}$ and $\lambda_{l m}$ are the amplitude and phase angle of a spherical harmonic term in Mars' gravitational field; $F_{l m p}(i)$ and $G_{l p q}(e)$ are polynomials dependent on the indices $l, m, p$, and $q$ (Kaula, 1966); and $\delta_{T}$ is the lag angle and $\sin 2 \delta_{T}$ is equal to $1 / Q$, the dissipation factor.

Commensurability other than 1:1 depends on either the eccentricity or inclination of Phobos. For 3:1, commensurability dependent on the inclination seems plausible: $l m p q=3310$, which results in

$$
k G \frac{m R^{5}}{r^{6}} \times \frac{3}{4} \frac{1}{Q}<\frac{G M^{*} R^{3}}{a^{4}}(f \sin i) J_{33}
$$

where $f$ is a factor from $F_{331}(i)$ of order $1 / 10$. Thence

$$
f J_{33} \sin i>k \frac{m}{M^{*}}\left(\frac{R}{a}\right)^{2} \frac{1}{Q}
$$

where $k$ is about $0.08 ; m / M^{*}, 10^{-9} ; a / R, 3 ;$ and $Q, 100$ to 1000 . Therefore

$$
J_{33} \sin i \gtrsim 10^{-13}
$$

$J_{33}$ would likely be of a magnitude intermediate between Earth's and the Moon's, say $3 \times 10^{-6}$. So the inclination $i$ of Phobos does not have to differ much from zero for this sort of coupling to occur.

The above term would be one of several terms dependent on either nonzero $i(p \neq 0)$ or nonzero $e(q \neq 0)$. As long as the commensurability is maintained, $a$ is unchanged, but $i$ and $e$ will continue to decrease. Hence the torque due to the combination of terms may pass through zero, and Phobos will slip out of the commensurability. Furthermore, if there is any significant tectonic activity in Mars, the $J_{l m}$ values will change.

HARTMANN: In analyzing the Mariner ' 69 photos of Martian craters, I have considered the question of whether we can account for Martian craters by considering only the present-day asteroids or whether we need to consider the early intense accretionary phase of bombardment by preasteroidal particles. There appear to be three marginal items of supporting evidence for the former:

(1) Craters from diameters of 50 to $200 \mathrm{~km}$ are not saturated on the Martian surface as would be expected if we could see all the way back to the time of accretion.

(2) This flat spot or undersaturation in the crater counts corresponds to asteroids in the diameter range of 6 to $25 \mathrm{~km}$, which is where the McDonald survey, the Palomar-Leiden survey (PLS), and the list of Mars crossers all show a flat spot in their diameter distributions. This flat spot divides what Kuiper has called the fragments from the larger original accretions. (See fig. D-1.) Thus, there is marginal evidence that we actually see the fossil imprint of the asteroid mass distribution when we look at Martian craters.

(3) Using Anders' calculated half-life for Mars-crossing asteroids and then calculating back $4 \times 10^{9} \mathrm{yr}$, we get approximately the observed number of Martian craters within a factor of 3 . This means that the present-day Mars-crossing population is approximately sufficient to account for the Martian craters without invoking the early accretionary bombardment.

According to this picture, Martian craters that we now see were caused by already-fragmented Mars-crossing asteroids. The initial mass distribution, such as discussed 


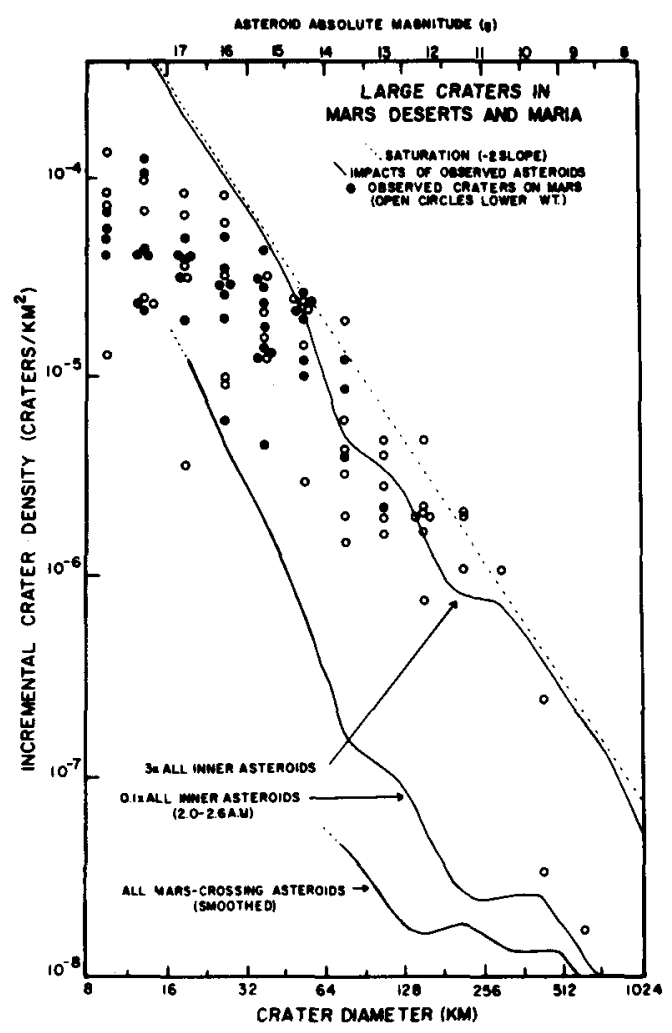

Figure D-1.-Diameter distribution of craters of Mars compared with diameter distribution predicted for craters formed from asteroids in the PLS (upper two solid curves) and present-day Mars-crossing asteroids (lowest curve). Upper solid and dashed curves show how saturation effects would truncate the Mars crater distribution. Nonetheless, the Mars crater counts from 50 to $200 \mathrm{~km}$ show a marginal trace of the "flat spot" in the asteroid size distribution, suggesting Mars craters are caused by impacts of Marscrossing asteroids over the last 4 aeons.

by Hills, ${ }^{1}$ had already been disturbed, which is reasonable because the original mass distribution probably would have been changed by fragmentation by the time Mars had accreted. It does leave one interesting point: We apparently do not see all the way back to the beginning of Mars' history (i.e., to its accretionary phase), as seems to be the case with the Moon.

LINDBLAD: Alfvén (Alfvén and Arrhenius, 1970) has suggested that an asteroidal belt may exist in the Jovian satellite system. By analogy with the formation of our planetary system it appears likely that an asteroid or "minor-moon" belt exists between the orbits of the Jovian satellites Amalthea (no. 5) and Io (no. 1). It is suggested that a special effort be made during a Grand Tour Jupiter flyby mission to detect such a belt, and measure parameters of interest; i.e., particle spatial density, size, and composition.

\footnotetext{
${ }^{1}$ See p. 225.
} 
A Jupiter-Saturn-Pluto mission with a Jupiter flyby trajectory passing about $100000 \mathrm{~km}$ inside the orbit of the satellite Io would be desirable for the Jovian "asteroid belt" experiment.

According to a recent JPL study, it is feasible to intercept the Jovian satellites Io, Ganymede, and Callisto in one single 1977 flyby mission. However, it is known that these satellites are rather similar in their physical characteristics. Alfvén (1971, private communication) has pointed out the desirability of including in the flight plan (if feasible) also a near encounter with one of the outer Jovian satellites (nos. 6, 7, and 10). It is suggested that feasibility studies for a Jupiter flyby mission including one of the outer Jovian satellites as well be considered.

A similar situation may exist in the Saturnian satellite system where a concentration of small particles may exist between satellites 5 and 6 .

\section{DISCUSSION REFERENCES}

Alfvén, H., and Arrhenius, G. 1970, Structure and Evolution of the Solar System. Astrophys. Space Sci. 8, 338.

Kaula, W. M. 1966, Theory of Satellite Geodesy. Blaisdell Pub. Co. Waltham, Mass.

Kuiper, G. P. 1956, On the Origin of the Satellites and the Trojans. Vistas in Astronomy (ed., Arthur Beer), vol. 2, pp. 1631-1666. Pergamon Press. New York. 